\title{
W-CDMA Adaptive Receiver with Space-Frequency Diversity for Multi-path Fading Channels
}

\author{
Alessandro Ciofini \\ LENST Electronic Telecommunication Department \\ University of Florence \\ Via S. Marta 3, 50139 Florence, Italy \\ ciofini@lenst.det.unifi.it \\ Romano Fantacci \\ LENST Electronic Telecommunication Department \\ University of Florence \\ Via S. Marta 3, 50139 Florence, Italy \\ fantacci@lenst.det.unifi.it \\ Luca Simone Ronga \\ CNIT Florence Unit \\ University of Florence \\ Via di S. Marta 3, 50139 Florence, Italy \\ luca.ronga@cnit.it
}

\begin{abstract}
Multiuser detection in severe multi-path fading environment is still under investigation. In this paper we propose a new receiver for up-link W-CDMA combining narrow-band sub-coding and adaptive antenna theory. Results have been compared to classic Rake recciver with omnidirectional antenna and they demonstrate how to significantly improve receiver's performance in terms of BER and algorithm complexity.
\end{abstract}

\section{Introduction}

In this paper a new receiver for terrestrial uplink UTRAN architecture oriented has been proposed and analyzed. Authors idea is to improve system's performance in terms of bit error rate, channel capacity, and so on combining two different approaches:

- sub-band coding

- spatial diversity

Traditional W-CDMA receivers, such as Rake receiver, use different replicas on frequency selective channels, but they also are affected by severe fading and AWGN corruption [3]. Using array antennas instead of omnidirectional ones and transmitting Q-PSK modulated CDMA signals over narrow-band frequency non selective channels we will demonstrate how to successfully improve uplink receiver's performance.

Adaptive beamforming has been also adopted to achive a better multiuser detection in urban environment without a perfect DOA's estimation. Referred system is an omnidirectional antenna with Rake receiver in a slow fading frequency selective channel. AWGN effect is also considered.

Sections are organized as follow:

System overview You can find here a full receiver's description in all its components.

Adaptive beamforming We briefly analyze adaptive beamforming theory and the adaptive algorithm we chose.

Performance evaluation A section completely dedicated to computer simulation results.

Conclusion Overall comments and possible future investigations.

\section{System overview}

As mentioned before, to reduce terrestrial mobile uplink signal corruption, we propose here a combined method for wide-band transmissions over a slow fading non selective channel with severe multi-path effect, such as a radio channel into urban environment [4].

You can see a complete receiver overview in fig. 2: the whole band used in W-CDMA transmission has been divided into four independent sub-bands, $W_{1}, W_{2}, W_{3}, W_{4}$ centered in $f_{1}, f_{2}, f_{3}, f_{4}$. Each one uses a shorter Gold sequence ${ }^{l}$ to spread the modulated signal. Users simply repeat

\footnotetext{
${ }^{1}$ We actually compared Orthogonal Gold sequences with length $L=$ 128 to $\mathrm{O}$-Gold with $L=32$ sequences.
} 

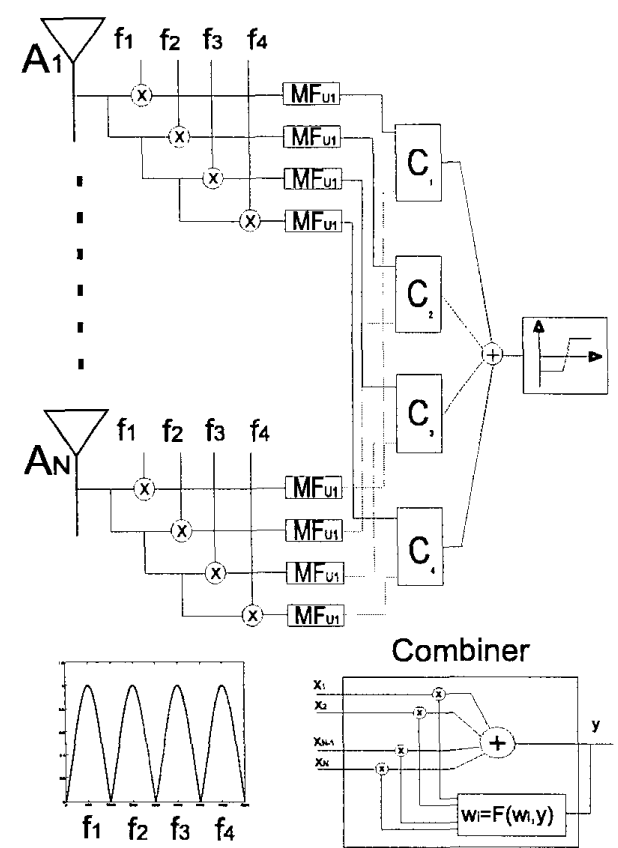

Figure 1. Receiver basic scheme

their bit streams four time, one each small band $\left(W_{i}=1024\right.$ $\mathrm{MHz}$, while $W_{T}=4096 \mathrm{MHz}$ ).

After channel corruption, an $\mathrm{N}$-dimension array, represented by $A_{1} \ldots A_{\mathrm{N}}$, is applied to receive $\mathrm{N}$ signals come from $\mathbf{M}$ different users. Signals are then frequency separated and processed by $\mathbf{N}$ matched filter to get back desired user bit stream.

While user's interferences, fading and AWGN effects are already part of the received signal, a combiner is then used to weight spatial replicas of desired user's signal.

Actually, two different solutions have been analyzed:

- maximum beamforming

- null beamforming

In the first case, array pattern is oriented to the desired user's position, known a priori, while the second technique puts $\mathbf{N}-1$ nulls toward the undesired users.

An adaptive algorithm can here be used to follow desired user during its movement, regardless its original position, that means weights can be time-variant.

We chose Constant Modulus algorithm (CMA), a blind adaptive technique, as described above. After array recombination, receiver simply adds different frequency replicas for the final decision.

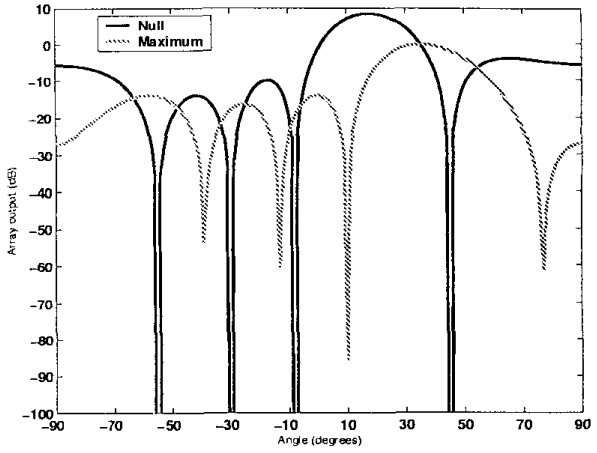

Figure 2. Linear 5-element array: two beamforming techniques.

\section{Adaptive beamforming in W-CDMA}

Digital beamforming and adaptive antennas are widely used and known since the last two decades. We focused our attention over an improved signal quality due to an intelligent use of spatial diversity to maximize desired user's signal or to minimize signals from others.

We suppose to use an uniformly spaced linear array, depicted with $\mathbf{N}$ identical isotropic elements.

Each element is weighted with a complex weight $V_{k}$, with $k=1 \ldots N$. The inter-element spacing is denoted by $d$ and the plane wave parameter are $\lambda$ (wave length) and $\theta$ (angle of arrival with respect to the array normal).

Adding all the element outputs together finds out the array factor $\mathcal{F}$, given by:

$$
\mathcal{F}(\theta)=\sum_{k=1}^{N} V_{k} \exp ^{i 2 \pi \frac{d}{\lambda} \cdot(k-1) \sin \theta}
$$

Let $\frac{d}{\lambda}=0.5$, if complex weight vector is:

$$
V_{k}=A_{k} \exp ^{i \cdot k \alpha}
$$

where $\alpha$ is the constant phase, then the array factor becomes:

$$
\mathcal{F}(\theta)=\sum_{k=1}^{N} V_{k} \exp ^{i \pi \cdot(k-1) \sin (\theta+\alpha)}
$$

From 3 , if $\alpha=-k \sin \hat{\theta}$, a maximum response of $\mathcal{F}(\theta)$ will result at $\theta=\hat{\theta}$. An example of five-element linear array is given in figure 3 , where five users have their DOA's according to the table 3 . Desired user is the first one.

Another set of weights can be found solving the linear system:

$$
\mathbf{T}^{\mathrm{H}} \cdot \mathbf{w}=\mathbf{v}
$$




\begin{tabular}{cc} 
Angle (degrees) & Distance (m) \\
\hline 35 & 200 \\
-55 & 100 \\
45 & 50 \\
-30 & 75 \\
-8 & 150
\end{tabular}

Table 1. User's initial position.

where $\mathbf{T}^{\mathrm{H}} \in \mathbb{C}^{M \times N}$ is the steering matrix

$$
\mathbf{T}_{l, m}=\exp ^{i \pi \cdot(l-1) \sin \left(\theta_{m}\right)} \text { for } l=1 \ldots N, m=1 \ldots M
$$

and $\mathbf{v} \in \mathbb{C}^{M}$ is that:

$$
\mathbf{v}_{k}= \begin{cases}1 & k=1 \\ 0 & \text { else }\end{cases}
$$

It should be noticed that has to be $M=N$ (i.e. number of users equal to array elements) to find the unique solution:

$$
\mathbf{w}=\left(\mathbf{T}^{\mathrm{H}}\right)^{-1} \cdot \mathbf{v}
$$

Figure 3 shows both techniques, reported as maximum and null: it should be noticed the different array response. The receiver described above uses the first set of weights, trying to lock the desired user, after interferences are significantly reduced by te gain process due to the spreading factor (see fig. 2 for details).

As illustrated in fig. 2, array can also use adaptive algorithms [1] to lock desired user during its movement. No matter what kind of initial pattern who choose, weights follow an adaptive criteria to match the pattern with the user to detect.

We propose Constant Modulus Algorithm (CMA), a blind adaptive beamforming technique widely known in literature [2]. Weights are updated as follow:

$$
\mathbf{w}_{n+1}=\mathbf{w}_{n}-\mu \cdot \mathbf{x}_{n} \cdot y_{n}\left(\left|y_{n}\right|^{2}-1\right)
$$

where $\mathbf{x}_{n} \in \mathbb{C}^{N}$ is the sensor array vector and $y_{n} \in \mathbb{C}$ the array output, after recombination due to the CMA weights

$$
y_{n}=\mathbf{w}_{n}{ }^{2} \cdot \mathbf{x}_{n}
$$

The only parameter in 8 is $\mu$, the convergence factor, we set to 0.001 , that assures both capture of desired user and algorithm convergence.

\section{Performance evaluation}

This section shows our results, obtained with a fiveelement linear array and five users: user's modulation is

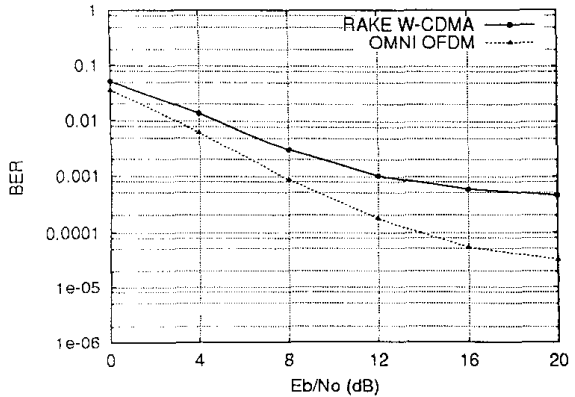

Figure 3. W-CDMA Rake vs. Omnidirectional antenna.

asynchronous Q-PSK with Gray coding, while channel model is GSM six-path wide-band channel model, urban environment [5].

Different scenarios have been analyzed:

- W-CDMA Rake receiver vs. omnidirectional antenna

- Omnidirectional antenna vs. static array

- Static array vs. adaptive array using CMA

Computer simulations has been realized using INeSiS, a new tool for numeric system modelling developed in $\mathrm{C}++$ at LENST $^{2}$ during last two years.

Figure 4 shows how narrow-band transmission works compared to W-CDMA. In this case, the system has been tested when $\mathbf{N}=1$ and $\mathbf{M}=5$, i.e., no arrays are used and the antenna becomes omnidirectional.

It is relevant to notice that receiver's performance in terms of bit error rate (BER) is better than traditional Rake receiver even though no spatial diversity is adopted: receiver, in fact, takes only advantage by frequency diversity due to four different transmitted replicas.

Starting from that, it is possible to improve receiver's performance using arrays instead of omnidirectional antenna.

Let $\mathbf{N}=\mathrm{M}$, i.e., arrays equal to users. In fig. 4 you can find two different results referred to different situations: the best case, where interferences are at least $2 \Theta_{3 \mathrm{~dB}}$ degrees away from desired user's direction, is compared with the worst case, where all interferences are within $\Theta_{3 \mathrm{~dB}}$, where $\Theta_{3 \mathrm{~dB}}$ is called the half power beam width (HPBW) and represent the angular width measured between the two points on the main lobe that are $3 \mathrm{~dB}$ below the peak of main lobe. For linear array uniform weighted $\Theta_{3 \mathrm{~dB}}$ is given by:

${ }^{2}$ Laboratorio di Elaborazione Numerica e Sistemi di Trasmissione, University of Florence, Italy. 


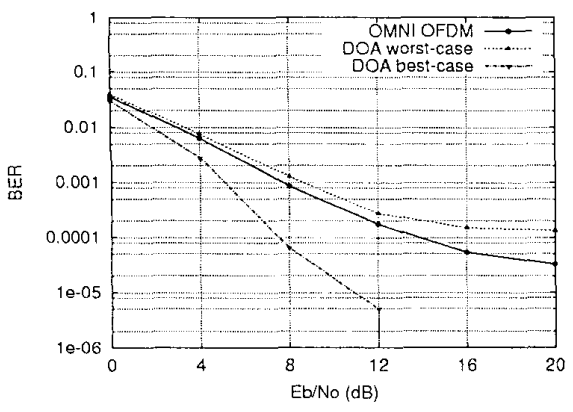

Figure 4. Omnidirectional antenna vs. static array

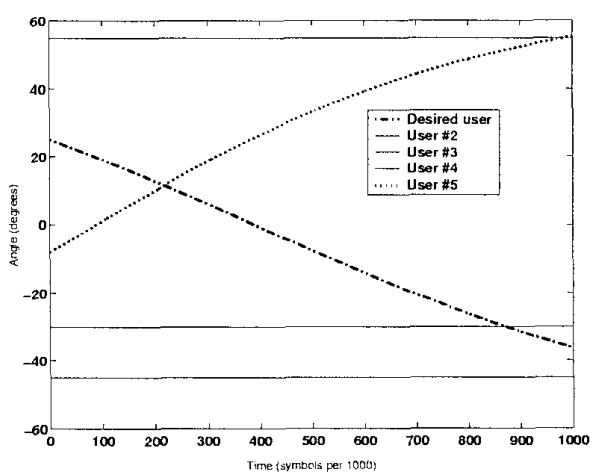

Figure 5. User's position during their movement.

$$
\Theta_{3 \mathrm{~dB}}=0.88 \cdot \frac{\lambda}{\mathrm{Nd}}
$$

From 10 , when $N=5$ and $\frac{d}{\lambda}=0.5$ you have $\Theta_{3 \mathrm{~dB}} \cong 20$ degrees: increasing $N$ means to reduce $\Theta_{3 \mathrm{~dB}}$.

Last step of our research was considering relative user's movement.

For the sake of conciseness, we suppose that only two users have initial non-zero velocity, included desired user, as shown in fig. 4.

Adaptive algorithm is now compared with static weighted array to evaluate mobile BER as time passing by. Figure 4 shows different results in terms of number of errors every 1000 symbols, while in fig. 4 you can directly evaluate initial and final antenna beam patterns.

It is easy to prove that adaptive CMA works better than static beamforming, despite CDMA technique can significantly reduce co-users interferences. Signal-to-noise ratio is set to $12 \mathrm{~dB}$.

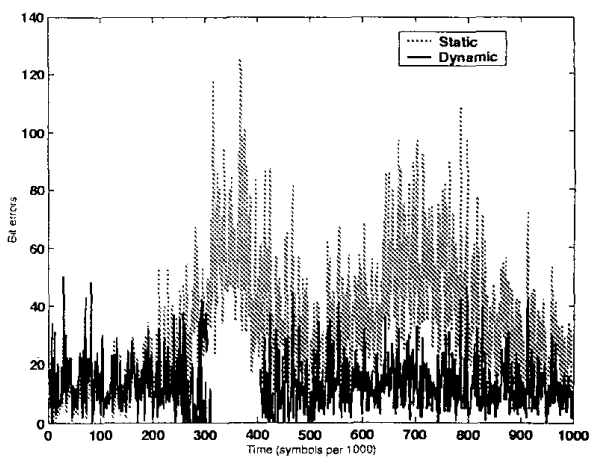

Figure 6. Mobile BER for static and dynamic beamforming with SNR $=12 \mathrm{~dB}$.

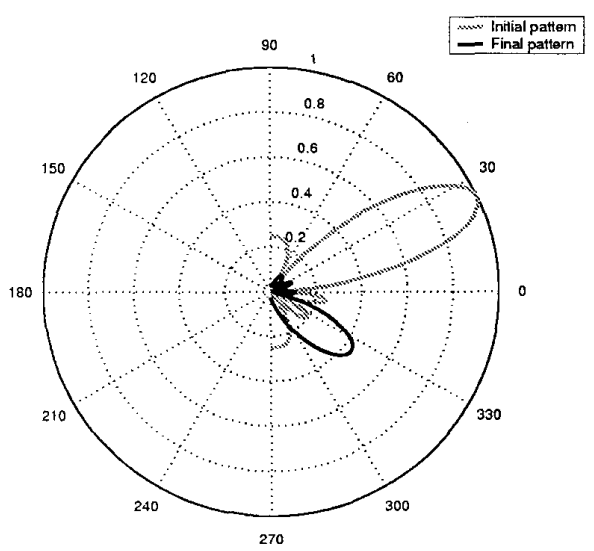

Figure 7. Final and initial array pattern.

Last we evaluate algorithm complexity compared with traditional RAKE receiver. Table 4 shows representative parameters.

\begin{tabular}{cc} 
Parameter & Description \\
\hline $\mathbf{L}$ & number of path \\
$\mathbf{N}$ & number of array \\
$\mathbf{S}$ & chip oversampling factor \\
$\mathbf{F}$ & number of frequency sub-channel \\
$\mathbf{G}$ & spreading factor \\
\hline
\end{tabular}

Table 2. Parameter definition.

We now can analyze algorithm complexity in terms of the number of complex multiplications and sums both receiver actually use to while working. 


\begin{tabular}{lr} 
RAKE. & $\mathrm{L} \cdot \mathrm{S} \cdot \mathrm{G}_{1}+\mathrm{S} \cdot \mathrm{G}_{1}$ \\
$\mathrm{CMA}$ & $\mathrm{F} \cdot \mathrm{N} \cdot \mathrm{S} \cdot \mathrm{G}_{\mathrm{F}}+\mathrm{F} \cdot \mathrm{N}-1+\mathrm{F}$ \\
\hline
\end{tabular}

Table 3. Number of complex multiplications.

\begin{tabular}{lr}
\hline RAKE. & $\mathrm{L}-1+\mathrm{S} \cdot\left(\mathrm{G}_{1}-1\right)$ \\
CMA & $\mathrm{F} \cdot \mathrm{N} \cdot \mathrm{S} \cdot\left(\mathrm{G}_{\mathrm{F}}-1\right)+\mathrm{F} \cdot(\mathrm{N}-1)+\mathrm{F}-1$ \\
\hline
\end{tabular}

Table 4. Number of complex sums.

Using tables 4 and 4 with $\mathbf{L}=6, \mathbf{N}=5, \mathbf{S}=1, \mathbf{F}=4$, $\mathbf{G}_{1}=128$ and $\mathbf{G}_{4}=32$, you obtain 132 sums and 896 multiplications for RAKE compared with 639 sums and 660 multiplications for proposed CMA-based receiver. We neglet weights update from global complexity calculation.

\section{Conclusion}

In this paper we proposed a new lechnique for W-CDMA receiver for up-link applications in UTRAN architecture. As already showed, our receiver has significantly improved classical Rake receiver's performance, in terms of bit error rate and overall algorithm complexity.

Future developments can further investigate over different array pattern adaptation, such as nullifying $N-1$ interference users to improve multiuser detection. Adaptive beamforming and sub-band has been demonstrated an efficient method against noise corruption, channel distortion due to multi-path phenomena and multi-user environment.

This work is conveyed out with the financial support of MURST.

\section{References}

[1] S. Haykin. Adaptive filter theory. Prentice Hall, 1996.

[2] T. K. L. J. Litva. Digital beamforming in wireless communications. Artech House Publishers, 1996.

[3] J. Proakis. Digital communications. McGraw-Hill, 1995.

[4] T. S. Rappaport. Wireless communications. Cambridge University Press, 1998.

[5] S. Verdù. Multiuser detection. Cambridge University Press, 1996. 